\title{
Beyond Tympanomastoidectomy: A Review of Less Common Postoperative Temporal Bone CT Findings
}

\author{
(D) A. Panda, (D) M.L. Carlson, (DF.E. Diehn, and (D).I. Lane
}

\begin{abstract}
SUMMARY: Postoperative temporal bone imaging after surgical procedures such as ossiculoplasty, tympanomastoidectomy, cochlear implantation, and vestibular schwannoma resection is often encountered in clinical neuroradiology practice. Less common otologic procedures can present diagnostic dilemmas, particularly if access to prior operative reports is not possible. Lack of familiarity with the less common surgical procedures and expected postoperative changes may render radiologic interpretation challenging. This review illustrates key imaging findings after surgery for Ménière disease, superior semicircular canal dehiscence, temporal encephalocele repairs, internal auditory canal decompression, active middle ear implants, jugular bulb and sigmoid sinus dehiscence repair, and petrous apicectomy.
\end{abstract}

ABBREVIATIONS: $\mathrm{AMEI}=$ active middle ear acoustic implants; ELS = endolymphatic sac; $\mathrm{LSC}=$ lateral semicircular canal; MCF = middle cranial fossa; PSC = posterior semicircular canal; SSC = superior semicircular canal; SSCD = superior semicircular canal dehiscence

M any neuroradiologists may be familiar with the imaging appearance of the middle and inner ear, internal auditory canal, and lateral skull base after common surgical procedures such as ossiculoplasty, tympanomastoidectomy, cochlear implantation, and vestibular schwannoma resection. ${ }^{1-3}$ Less common otologic procedures can present diagnostic dilemmas, particularly if access to prior operative reports is lacking. Neuroradiologists may not be aware of these surgical procedures either due to the decreasing numbers of these surgeries performed today or some of the newer surgeries confined to specialized academic centers and thus less commonly encountered in routine practice. These postoperative temporal bone findings can have unique imaging features based on the type of surgical procedure and indication. Lack of familiarity with these surgical procedures and expected postoperative changes may render radiologic interpretation challenging. The purpose of this image-rich review is to illustrate less common postoperative temporal bone CT findings that neuroradiologists may encounter and would ideally be able to recognize and to differentiate from disease

Received May 4, 2020; accepted after revision July 18.

From the Departments of Radiology (A.P., J.I.L., F.E.D.) and Otolaryngology-Head and Neck Surgery (M.L.C.), Mayo Clinic, Rochester, Minnesota.

Paper previously presented as an Educational Exhibit at: Annual Meeting of the Radiological Society of North America, December 1-6, 2019; Chicago, Illinois, and was awarded a Certificate of Merit.

Please address correspondence to John I. Lane, MD, Department of Radiology, Mayo Clinic, 200 1st St SW, Rochester, MN 55905; e-mail: lane.john@mayo.edu; @AnanyaPanda15; @MatthewLCarlsol; @MayoRadiology

-- Indicates open access to non-subscribers at www.ajnr.org

http://dx.doi.org/10.3174/ajnr.A6802 mimics. This review illustrates imaging findings after surgery for Ménière disease, superior semicircular canal dehiscence, temporal encephalocele repairs, internal auditory canal decompression, active middle ear implants, jugular bulb and sigmoid sinus dehiscence repair, and petrous apicectomy.

\section{Ménière Disease}

Ménière disease is characterized by recurrent attacks of episodic vertigo, fluctuating low-frequency sensorineural hearing loss, tinnitus, and aural fullness. The pathophysiology is attributed to endolymphatic hydrops, a histologic finding in which the endolymphatic compartment including the scala media is dilated. ${ }^{4}$ Endolymph is the fluid within the membranous labyrinth, including the vestibule, semicircular canals, and scala media of the cochlea. Although the physiology is debated, the endolymphatic sac (ELS) is thought to maintain hydrostatic pressure and endolymph homeostasis for the inner ear, and its dysfunction may contribute to the pathophysiology of this disease. ${ }^{5}$ While many patients respond well to lifestyle changes such as low-salt diet and medical management with diuretics and betahistine, $>20 \%$ of patients develop intractable Ménière disease. Treatment options for these patients with medically refractory symptoms include intratympanic corticosteroid injection, gentamicin injection, and surgery. Surgical treatment may be classified as "nonablative" (in which inner ear function is preserved), including endolymphatic sac decompression, or "ablative" (in which inner ear function is lost), including labryinthectomy and vestibular neurectomy. ${ }^{6}$

ELS surgery, ie, either ELS decompression or ELS shunting, is a nonablative surgical option with moderate efficacy and little 

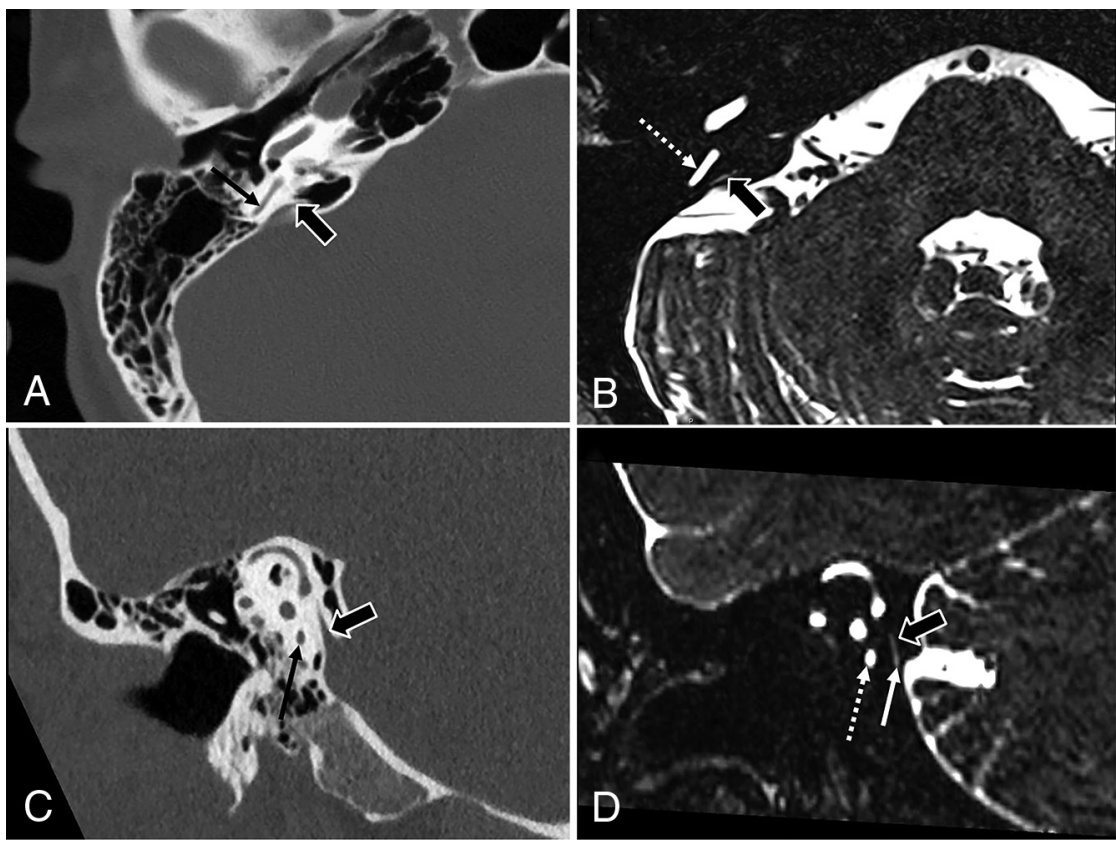

FIG 1. Anatomy of the endolymphatic duct and sac on axial ( $A$ and $B)$ and Pöschl view ( $C$ and $D)$ $C T$ and 3D fast spin-echo T2-weighted MR images. The endolymphatic duct connects with the endolymphatic sac. Both structures are not directly seen on CT, but CT shows the osseous vestibular aqueduct that houses the endolymphatic duct (block arrows, $A$ and $C$ ). Note the location of vestibular aqueduct extending to the cortex of the posterior temporal bone (block arrow, $A$ ). On MR imaging, the endolymphatic duct can be seen directly as a T2WI hyperintense tubular structure (block arrows, $B$ and $D$ ), extending inferiorly beneath the dura of the posterior cranial fossa at the expected location of endolymphatic sac (white arrow, D). Note the close proximity of the posterior semicircular canal (black arrows on CT and dotted white arrow on MR imaging), an important anatomic landmark to identify the endolymphatic sac intraoperatively.

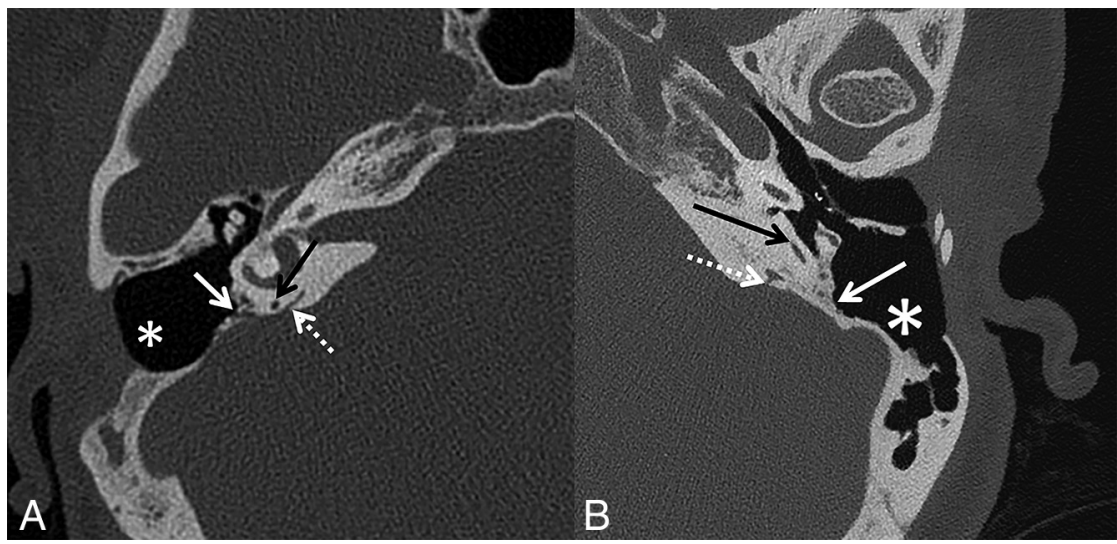

FIG 2. Endolymphatic sac decompression ( $A$ ) versus mastoidectomy (B). A, Transmastoid endolymphatic sac shunt surgery: Postoperative changes of right canal wall-up mastoidectomy (asterisk). The posterior margins of the mastoidectomy defect extend up to the presigmoid osseous plate at the expected location of the endolymphatic sac (solid white arrow shows the location of transmastoid endolymphatic sac shunting). Removal of the bone overlying the posterior semicircular canal (black arrow) and vestibular aqueduct (dotted white arrow) indicates good surgical exposure of the endolymphatic sac. The silastic shunt is not directly visualized on CT. B, For comparison, an image from another patient with a simple canal wall-up mastoidectomy and ossiculoplasty for chronic otitis media. Note the mastoidectomy defect (asterisk) and the intact posterior cortex (solid white arrow) overlying the posterior semicircular canal (black arrow) and vestibular aqueduct (dotted white arrow). risk to hearing. ${ }^{5,6}$ The ELS is not directly visualized on CT imaging; one can, however, see the osseous margins of the vestibular aqueduct, which encase the membranous endolymphatic duct. On MR imaging, the endolymphatic duct that opens into the ELS at the operculum can be readily identified as a T2WI hyperintense structure. The location of the ELS is inferred at the terminal end of the endolymphatic duct at the dura of the posterior cranial fossa and just behind the posterior semicircular canal (PSC) (Fig 1). ${ }^{7}$ The Donaldson line is a useful surgical landmark to identify the ELS during ELS decompression. This line is drawn along the long axis of the lateral semicircular canal (LSC) bisecting the PSC. The estimated location of the ELS is just anterior and inferior to the junction of this line and the sigmoid sinus. The surgical steps of ELS decompression comprise a wide canal wall-up mastoidectomy with decompression of the sigmoid sinus and the presigmoid dura, often referred to as the Trautman triangle. The ELS is identified as a thickening of dura mater posterior to the PSC and is sub sequently widely decompressed without inadvertent durotomy. Additional shunt placement is optional and has largely fallen by the wayside. ${ }^{8,9}$ On postoperative imaging, surgical changes of ELS decompression comprise of canal wallup mastoidectomy, which further extends up to the posterior osseous cortex with presigmoid dura decompression. Removal of the bone immediately posterior and inferior to the PSC and vestibular aqueduct indicates good surgical exposure of the ELS. Identifying the posterior extent of the mastoidectomy margin and exposure of the presigmoid dura differentiates this procedure from a simple cortical mastoidectomy (Fig 2).

While no longer performed today, sacculotomy via the Cody tack procedure is a surgery for Ménière disease that was initially popularized in the early 1970s. The premise behind the procedure was that an internal shunt 
between the endolymphatic and perilymphatic compartments may alleviate excess pressure within the membranous labyrinth. Surgically, a prosthetic tack is introduced through the footplate of the stapes that will puncture the saccule whenever saccular dilation occurs, thereby providing "automatic repetitive sacculotomy" (Fig 3). ${ }^{10}$ On CT, this should not be mistaken for vestibular migration of an ossicular prosthesis, most commonly a stapes prosthesis. The stapes is normal and intact in patients with sacculotomy hardware, in contradistinction to those with migrated stapes prostheses after stapedectomy procedures for otosclerosis (Fig 3).

Surgical labyrinthectomy is performed for intractable Ménière disease after exhaustion of more conservative nonablative procedures and in patients with poor residual hearing. The goal of labyrinthectomy is to destroy the labyrinthine neuroepithelium and thereby eliminate faulty vestibular stimuli from the diseased ear. ${ }^{11}$ Several surgical approaches can be used to perform labyrinthectomy. Some authors advocate a transcanal approach through the external auditory canal, whereby the incus and stapes are removed to access the vestibule. More commonly, the transmastoid approach is used, in which a canal wall-up mastoidectomy is performed, the 3 semicircular canals are exenterated, and the vestibule is cleaned of neuroepithelium. ${ }^{12,13}$ Surgical labyrinthectomy for any indication may lead to labyrinthitis ossificans and, much less commonly, CSF leak or facial nerve injury. In patients with surgical labyrinthectomy, air in the labyrinth is considered an expected finding; therefore, these cases should not be mistaken for traumatic or other acquired causes of perilymph fistula or pneumolabyrinth (Fig 4).

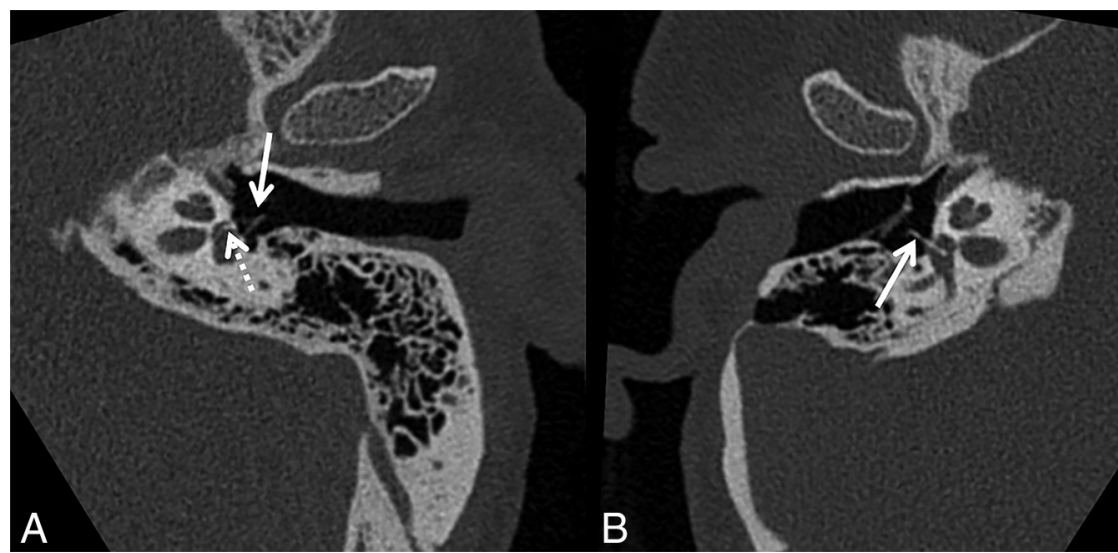

FIG 3. Sacculotomy $(A)$ versus stapes prosthesis migration $(B)$. A, Sacculotomy with insertion of a Cody tack prosthesis. Oblique CT image reformatted along the long axis of the stapes $(A)$ shows a linear metallic device in the saccule (dotted white arrow). This was a Cody tack device for a sacculotomy. Note the intact stapes (solid white arrow). $B$, Vestibular migration of stapes prosthesis. Oblique $C T$ image reformatted along the long axis of the stapes $(D)$ shows that the stapes suprastructure is replaced by a prosthesis (solid white arrow), with intrusion of the prosthesis into the vestibule.

\section{Semicircular Canal Dehiscence}

Superior semicircular canal dehiscence (SSCD) is characterized by thinning of the osseous covering overlying the superior semicircular canal. Due to the dehiscence, there is a direct communication between membranous labyrinth and the overlying dura or superior petrosal sinus, leading to a "third window phenomenon," ie, abnormal dissipation of sound/pressure waves through the dehiscence. Clinically, a third window phenomenon is characterized by various signs such autophony (ie, prominently hearing one's own voice in the affected ear), sound- or pressure-induced vertigo and/or nystagmus (Tullio phenomenon and the Hennerbert sign, respectively), pulsatile tinnitus, and low-frequency conductive hearing loss with negative bone-conduction thresholds. ${ }^{13,14}$ The

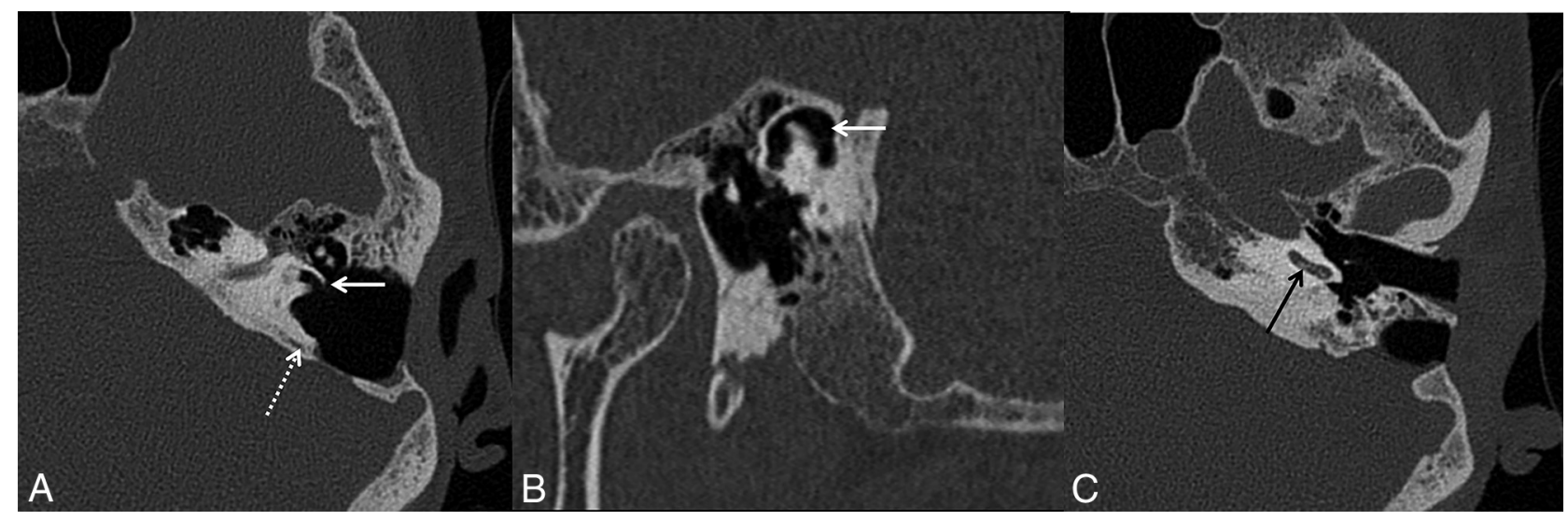

FIG 4. Surgical labyrinthectomy with cochlear labyrinthitis ossificans. Axial CT image $(A)$ of the left temporal bone shows changes of prior transmastoid endolymphatic sac surgery (dotted white arrow), with a mastoidectomy defect extending posteriorly to the expected location of the endolymphatic sac. Postoperative changes of the subsequently performed hearing-ablative surgical labyrinthectomy are seen as surgical removal of bone overlying the lateral semicircular canal with a fistulous connection between the lateral semicircular canal and the mastoid defect (solid white arrow, A). Air also extends to the superior semicircular canal (solid white arrow, $B$ ). A more caudal image $(C)$ shows mineralization within the basal turn of the cochlea (black arrow, C), consistent with labyrinthitis ossificans, an expected postoperative finding following labyrinthectomy. 


\begin{tabular}{|c|c|c|c|}
\hline Approaches & Advantages & Disadvantages & $\begin{array}{c}\text { Surgery Technique } \\
\text { Approach Most Suitable for }\end{array}$ \\
\hline $\begin{array}{l}\text { Middle Cranial } \\
\text { Fossa }\end{array}$ & $\begin{array}{l}\text { Direct visualization of } \\
\text { dehiscence }\end{array}$ & $\begin{array}{l}\text { More invasive requiring craniotomy } \\
\text { and limited temporal lobe } \\
\text { retraction }\end{array}$ & $\begin{array}{l}\text { All kinds of SSC repair: canal resurfacing, } \\
\text { plugging, capping }\end{array}$ \\
\hline Transmastoid & $\begin{array}{l}\text { Less invasive, standard } \\
\text { mastoidectomy } \\
\text { approach }\end{array}$ & No direct visualization of dehiscence & $\begin{array}{l}\text { Canal plugging and modified resurfacing (of SSC } \\
\text { sidewalls and not directly over dehiscence) }\end{array}$ \\
\hline Transcanal & Outpatient setting & Less efficacious in long-term & Reinforcement or occlusion of round window \\
\hline
\end{tabular}

${ }^{a}$ Modified with permission from Mau et al, ${ }^{16}$ Ward et al, ${ }^{17}$ and Succar et al. ${ }^{19}$

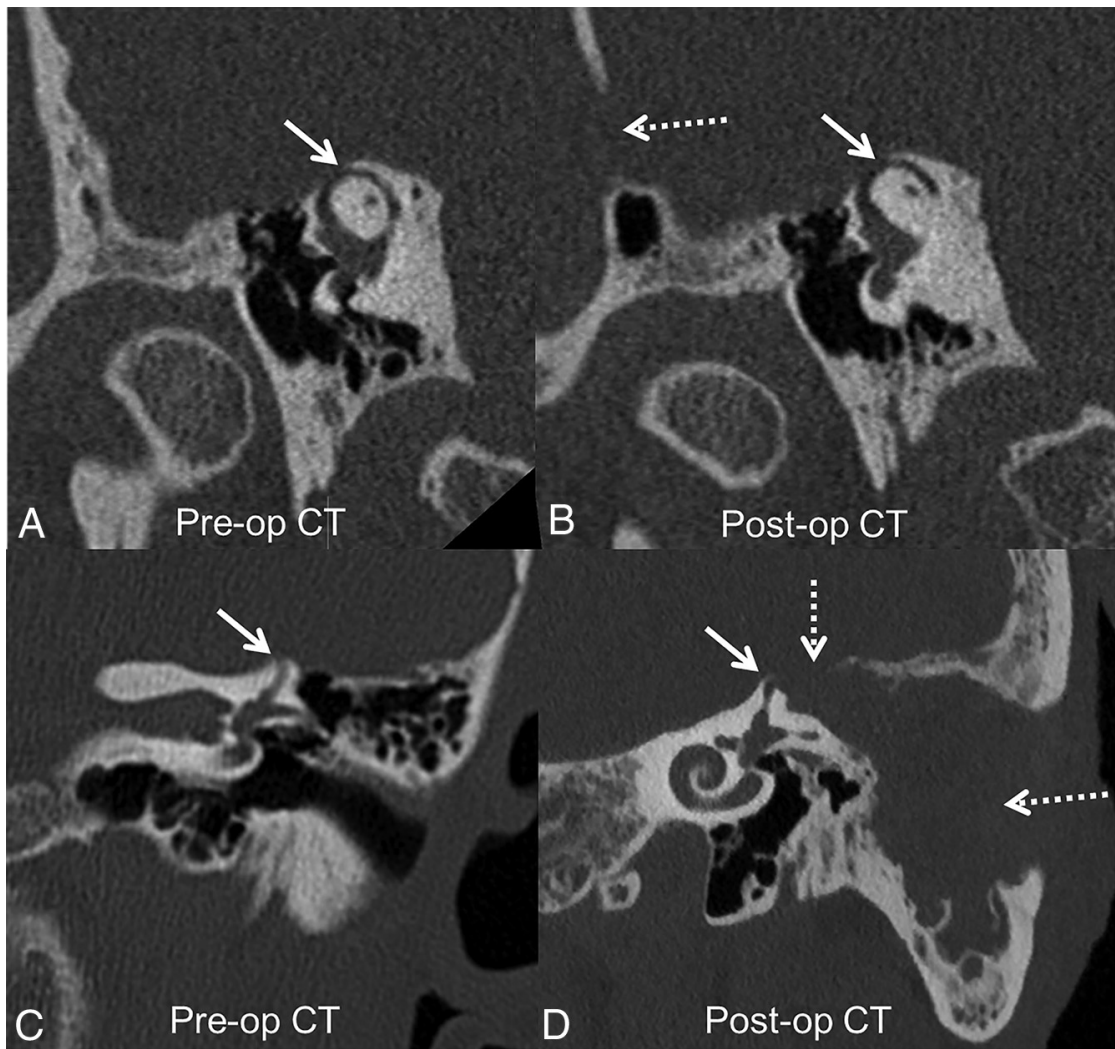

FIG 5. Superior semicircular canal dehiscence repair through a middle cranial fossa approach $(A$ and $B)$ and transmastoid approach $(C$ and $D)$. Preoperative Pöschl view $(A)$ shows dehiscence of the mid-third of the left superior semicircular canal (solid white arrow). A middle cranial fossa approach (dotted white arrow, $B$ ) was used to plug the superior semicircular canal with bone wax and temporalis fascia. Postoperative CT (B) shows further loss of bone overlying the superior semicircular canal due to drilling of bone overlying the superior semicircular canal to plug it (solid white arrow). Bone wax and temporalis fascia are radiolucent and not directly seen on CT. Postoperative changes of middle cranial fossa repair can be missed on temporal bone CT if not specifically sought and in the absence of clinical details. Preoperative CT (C) shows a large left superior semicircular canal dehiscence (solid white arrow). A transmastoid approach (dotted white arrows, $D$ ) was used to access the roof of the superior semicircular canal and plug it with bone wax and temporalis fascia. There is expected soft tissue in the mastoidectomy defect without evidence of otitis media (not shown). Pre-op indicates preoperative; Post-op, postoperative.

reformatted parallel to the long axis of petrous bone), SSCD is characterized by parallel or diverging walls of the SSC as they approach the floor of the middle cranial fossa (MCF). ${ }^{15}$

Surgeries for SSCD are directed toward repair of the dehiscence by canal plugging, resurfacing, or combinations thereof. ${ }^{16}$ Both transmastoid and MCF approaches have been described with a $>90 \%$ success rate (Table). ${ }^{17,18}$ Examples of materials used include bone wax, bone chips, and temporalis fascia for canal plugging and cartilage, bone, temporalis fascia, and hydroxyapatite bone cement for resurfacing. ${ }^{17}$ Most patients do not need imaging after SSCD repair. CT or MR imaging is typically performed in those patients with persistent vertigo or suspected contralateral dehiscence. ${ }^{15}$ Postoperative imaging findings differ on the basis of the material used and the procedure performed. If the SSC has been plugged with bone cement or if there is secondary labyrinthitis ossificans, the occluded semicircular canal segment may appear hyperattenuating on CT. However, both bone wax and fascia are radiolucent materials and not directly visualized on CT. Thus, on postoperative CT, there may be persistent or greater thinning of bone overlying the SSC. This should not be mistaken for persistent SSCD or surgical failure if the patient is asymptomatic (Fig 5). Compared with CT, MR imaging can better depict SSC plugging by loss of T2WI hyperintensity within the semicircular canal. Other postoperative findings may include temporal lobe

diagnosis of SSCD can be suggested on heavily T2-weighted MR images but is best made on high-resolution axial and oblique reformatted CT views. On the Pöschl view (ie, images reformatted perpendicular to the long axis of petrous bone), SSCD is characterized by an absent or thinned-out osseous covering overlying the superior semicircular canal (SSC). On the Stenvers view (ie, images encephalomalacia due to retraction of the temporal lobe during the MCF approach. ${ }^{15}$

Round window occlusion is another minimally invasive approach for semicircular canal dehiscence treatment. This is performed through a transcanal approach and can be done as an outpatient surgery. Occluding or reinforcing the round window 
with autologous material can lead to symptom improvement by eliminating the third window effect, but it may also contribute to a conductive hearing loss. Although this surgery is a less-invasive treatment option for these patients, it has less efficacy compared with direct dehiscence repair. ${ }^{19}$ Postoperative imaging shows soft tissue obliterating the normal air-filled round window niche (Fig

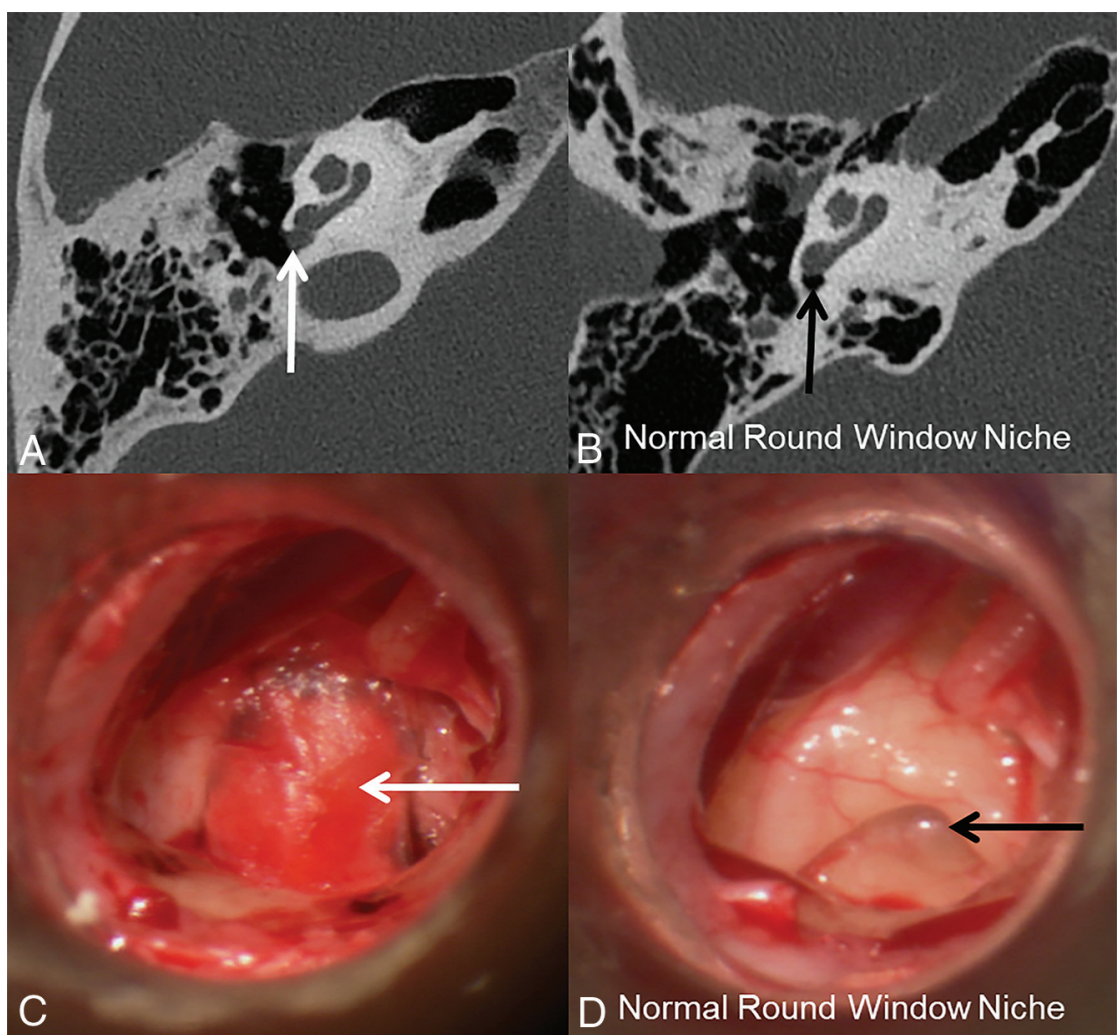

FIG 6. Transcanal occlusion of the round window for superior semicircular canal dehiscence. A patient with bilateral superior semicircular canal dehiscence underwent bilateral transcanal occlusion of the round windows with cartilage graft and fascia. Postoperative axial CT image of the right temporal bone $(A)$ shows nodular soft tissue in the round window niche (white arrow). A normal round window niche is air-filled compared with that of a healthy patient (black arrow, $B$ ). Intraoperative image (C) shows a round window after occlusion with fascia/cartilage graft (white arrow), compared with that of a healthy patient (black arrow, D).
6). This should not be mistaken for middle ear disease such as glomus tympanicum or cholesteatoma on imaging. Occlusion of the round window is also offered to patients with a localized perilymph fistula (spontaneous, traumatic, or secondary to erosive middle ear disease). ${ }^{20}$ This can look similar to round window occlusion for semicircular canal dehiscence and again should not be mistaken for middle ear disease.

Isolated lateral semicircular canal dehiscence is rare. Defects in the LSC are more likely due to erosion secondary to infectious, inflammatory, or neoplastic conditions. ${ }^{13}$ Very rarely, a radiologist may encounter a patient who underwent a remote LSC fenestration for otosclerosis. In LSC fenestration, an opening is made in the lateral semicircular canal through the mastoid; imaging findings can be mistaken for lateral semicircular canal dehiscence or LSC fistula if one is not aware of this procedure (Fig 7). Because this procedure is now replaced by more effective stapedotomy/stapedectomy procedures, these imaging findings are expected to be found only in patients with remote surgeries.

\section{Temporal Encephaloceles and CSF Leak}

Temporal meningoceles or encephaloceles secondary to skull base dehiscence may be congenital, posttraumatic, iatrogenic, or secondary to idiopathic intracranial hypertension (pseudotumor cerebri). ${ }^{21}$ Studies have shown an association between SSCD and temporal meningoencephaloceles in patients with idiopathic intracranial hypertension. $^{22}$ The pathophysiology of both

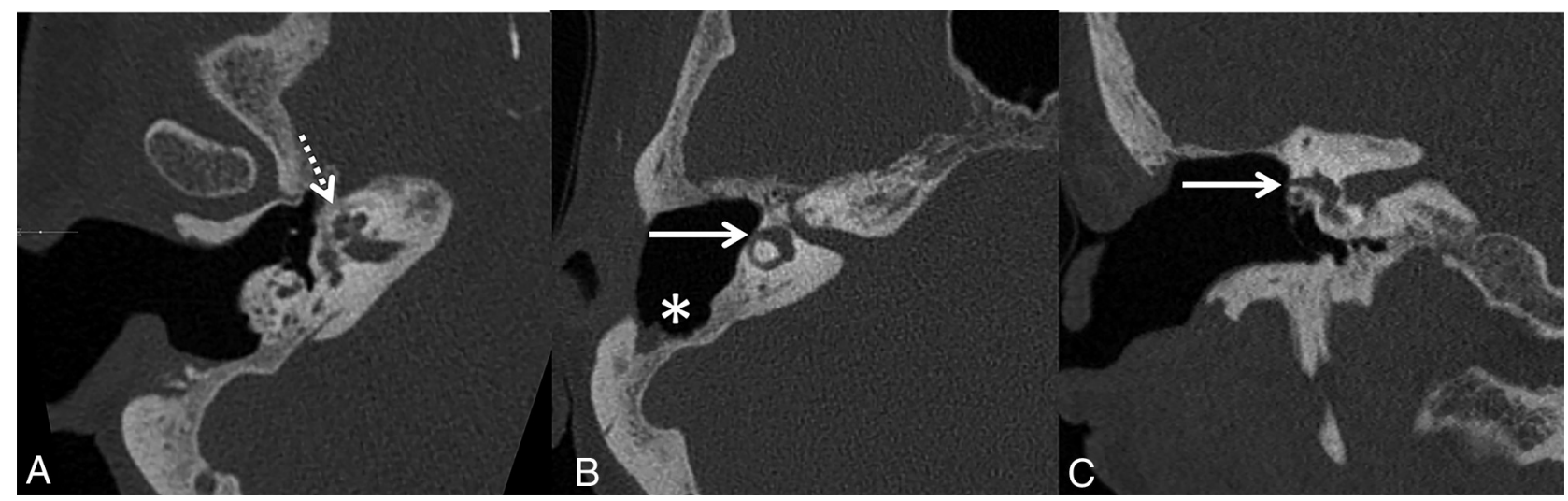

FIG 7. Postoperative changes of lateral semicircular canal fenestration performed for otosclerosis. Axial CT image shows lucency around the cochlea (the "double ring" sign) (dotted white arrow, $A$ ) consistent with retrofenestral otosclerosis. Postoperative axial (B) and coronal (C) CT images show an osseous defect over the lateral margin of lateral semicircular canal (solid white arrows) due to surgical fenestration of the lateral semicircular canal. This should not be mistaken for a lateral semicircular canal fistula. Note the associated mastoidectomy defect (asterisk). 


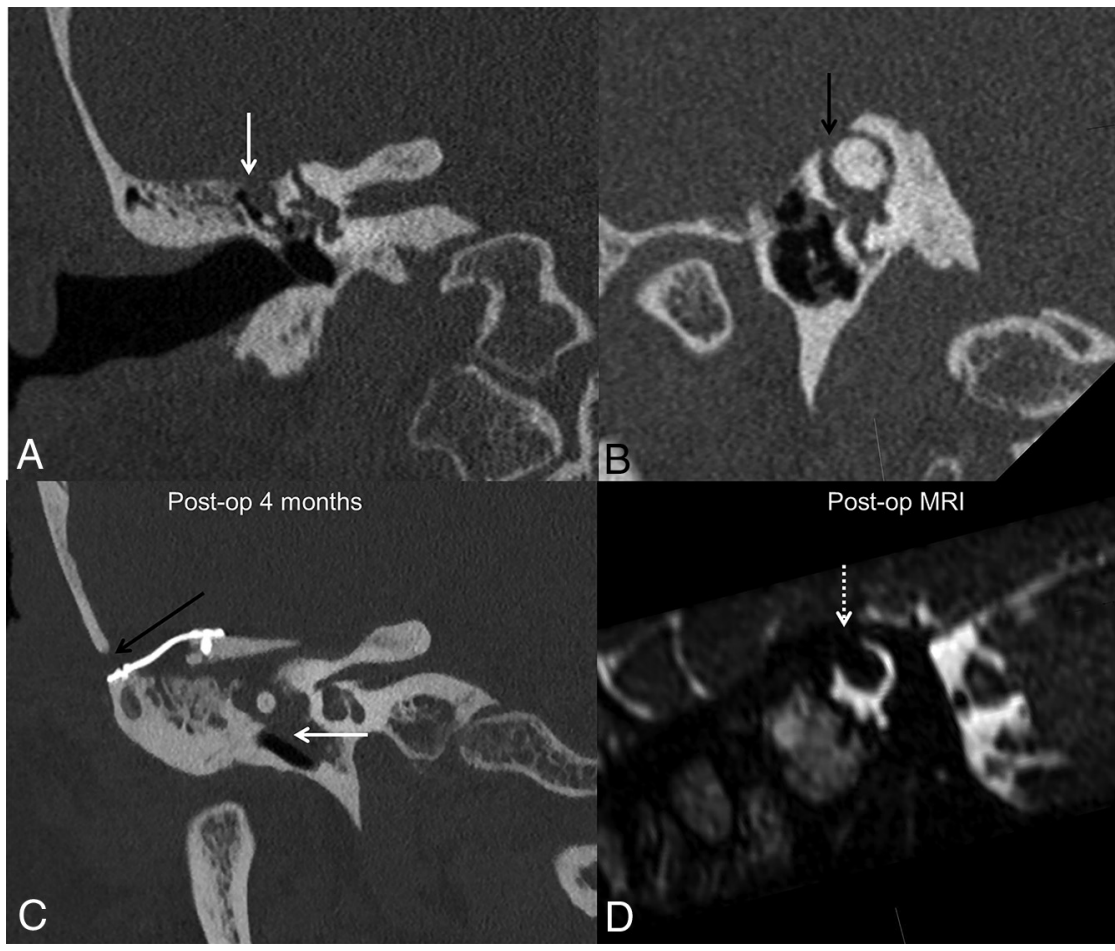

FIG 8. Temporal meningoencephalocele repair and superior semicircular canal dehiscence plugging complicated by CSF leak in a patient with idiopathic intracranial hypertension. Preoperative coronal CT of right temporal bone $(A)$ shows a defect in the tegmen tympani. Soft tissue extends through the defect into the right middle ear (solid white arrow, A). Pöschl view (B) shows dehiscence of the mid-portion of the right superior semicircular canal (black arrow, B). Postoperative images show changes of right middle canal fossa repair with the bone and fascia graft held down with plate screws (black arrow, C). The right SSC was plugged with bone wax in the same setting, and the roof was resurfaced with fascia graft, better appreciated on the heavily T2-weighted MR imaging $(D)$, which shows the expected focal loss of fluid signal in the right SSC at the site of plugging (dotted white arrow, D). Postoperatively, the patient developed a CSF leak requiring additional surgical repair (solid white arrow, C). The patient also had a small encephalocele on the left side (not shown) consistent with intracranial hypertension. Post-op indicates postoperative.

to auditory, vestibular, or facial nerve dysfunction. Craniometaphyseal dysplasia and Camurati-Engelmann disease (progressive diaphyseal dysplasia) are two such examples that may necessitate internal auditory canal decompression due to seventh and eighth cranial nerve deficits. ${ }^{24,25}$ Compressive effects may also be noted on other skull base foramina, cranial nerves, vessels, and brain stem. In these patients, internal auditory canal decompression is performed through the MCF approach to remove the superior and the anterior portions of the petrous bone. On postoperative imaging, one should evaluate the adequacy of internal auditory canal decompression, which should ideally include the labyrinthine segment of the facial nerve, and ensure that the arcuate eminence (the bone overlying the SSC) is intact (Fig 9). Unfortunately, longterm follow-up may show heterotopic or dystrophic ossification, necessitating revision decompression surgeries.

\section{Implantable Active Middle Ear Acoustic Implants}

Implantable active middle ear acoustic implants (AMEI) are alternatives to hearing aids in appropriately selected patients. These may be offered to patients with variable severity of conductive, sensorineural, or mixed hearing loss who cannot wear hearing aids due to infection or defects or atresia of

meningoencephaloceles and SSCD is attributed to the thinning of the skull base and the roof of the SSC due to raised intracranial pressure. Raised intracranial pressure can also lead to CSF leaks. ${ }^{13}$ Surgical approaches for skull base dehiscence repairs include transmastoid and MCF approaches. The transmastoid approach is preferred for smaller defects and defects within the tegmen mastoideum, while the MCF approach is used for defects that are larger, multiple, or in the tegmen epitympanum or petrous apex. ${ }^{23}$ Repair materials most commonly include fascia and bone grafts and, less commonly, bone cement. In those patients with concomitant SSCD, dehiscence repair can be performed at the time of meningoencephalocele repair (Fig 8). It is also recommended to look for meningoencephaloceles and SSCD on the contralateral side because bilateral involvement is relatively common in patients with idiopathic intracranial hypertension. ${ }^{21}$

\section{Internal Auditory Canal Decompression}

Internal auditory canal decompression is commonly performed for vestibular schwannoma to access the tumor but may also be used to treat osseous dysplasias of the temporal bone that are associated with progressive narrowing of the internal auditory canals leading the external auditory canal or who do not wish to wear hearing aids for cosmesis. ${ }^{26,27}$ These implants transduce acoustic sound energy to the cochlear perilymph either through skull vibration via the temporal bone cortex or via the ossicular chain. These differ from passive middle ear implants such as an ossicular reconstruction prosthesis. They also differ from cochlear implants or auditory brain stem implants that directly simulate the cochlear nerve or brain stem nuclei, respectively. ${ }^{28}$ There are a variety of AMEIs available, some of which are FDA approved in the United States while others are available in Europe. ${ }^{26}$ From an imaging perspective, it is important for radiologists not to mistake AMEIs for cochlear implants on postoperative CT. A primary distinguishing feature is that a cochlear implant will have an electrode within the cochlear lumen that typically extends to a minimum angular depth of $270^{\circ}$ to $360^{\circ}$, whereas AMEIs will not have an intracochlear component. Similar to earlier cochlear implant designs, many AMEIs are not MR imaging conditional.

AMEIs can either be partially implantable or fully implantable. ${ }^{28}$ Partially implantable AMEIs have an external sound processor and an internal floating mass transducer. Partially implantable AMEIs can either have a fully external sound processor held by a magnet 


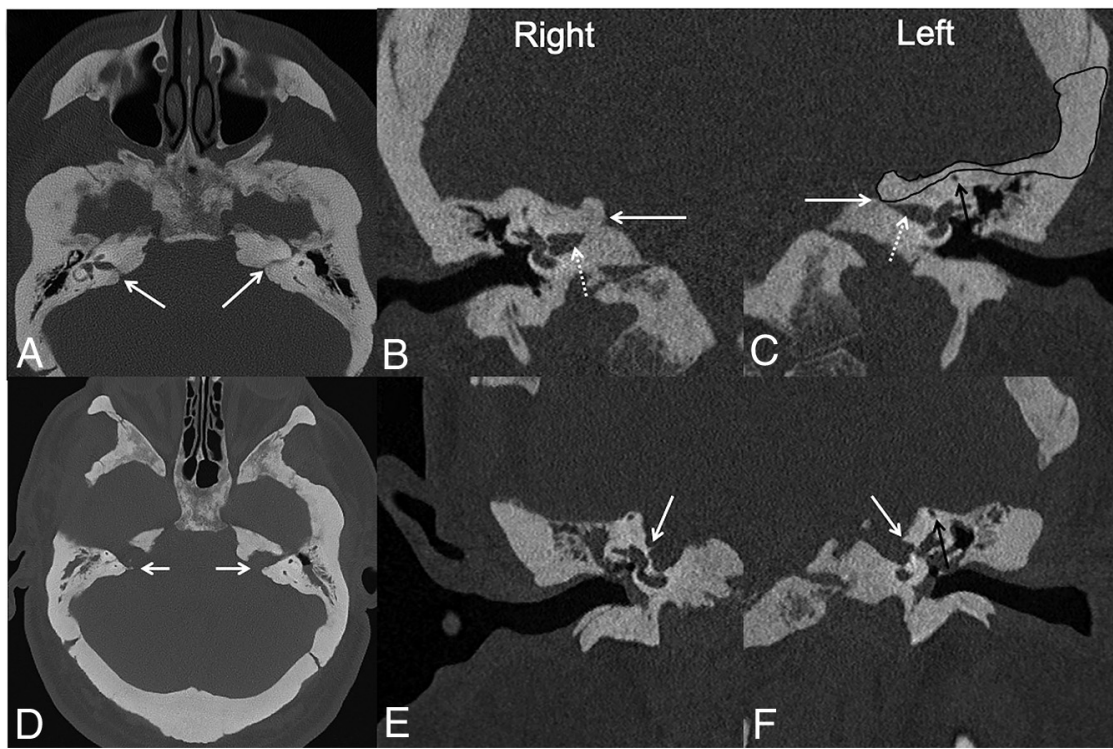

FIG 9. Internal auditory canal decompression for Camurati-Engelmann disease. Upper row: Preoperative $\mathrm{CT}$ images $(A-C)$ in a patient with Camurati-Engelmann disease show extensive osseous skull base thickening and near-complete obliteration of both internal auditory canals (left $>$ right) (solid white arrows, $A$ ). Coronal images ( $B$ and $C$ ) show marked narrowing of both internal auditory canals (dotted white arrows), more so medially where the porus acousticus is nearly obliterated due to hypertrophic osseous overgrowth of the walls of internal auditory canals (solid white arrows). Lower row: Postoperative CT images (D-F) show widely patent internal auditory canals bilaterally (solid white arrows, $D$ ). Coronal images ( $E$ and $F$ ) show decompression of the internal auditory canals by removal of the superior and anterior portions of the petrous bones (solid white arrows). The black polygon in C depicts the portion of the bone removed through the middle cranial fossa approach to decompress the internal auditory canal. Care is taken to preserve the arcuate eminence over the superior semicircular canal (black arrows, $C$ and $F$ ). (eg, Vibrant Soundbridge; Med-EL) or an in-the-canal processor (eg, Maxum; Ototronix) (Fig 10). ${ }^{28}$ The floating mass transducer can be crimped to the long process of the incus or the stapes footplate or suprastructure or coupled to the round window. The floating mass transducer is stimulated by the processor, which, in turn, creates a mechanical vibration that results in perilymph fluid movement within the cochlea. ${ }^{29}$ Thus, for proper functioning, the implant must be correctly coupled to the ossicular system or round window. Any break in the coupling of the floating mass transducer leads to device malfunction. The goal of postoperative imaging in these patients is to identify the device components and the integrity of the coupling system (Fig 11).

In fully-implantable AMEIs (eg, Esteem; Envoy), the processor is internal and secured to the mastoid bone. It has a sensor coupled to the body of the incus and a driver coupled to the stapes. $^{30}$ The incudostapedial joint is disarticulated at the time of device implantation to separate the sensor and the driver functions. Thus, incudostapedial disarticulation on postoperative CT is expected and should not be interpreted as pathologic decoupling or discontinuity (Fig 12). ${ }^{28}$

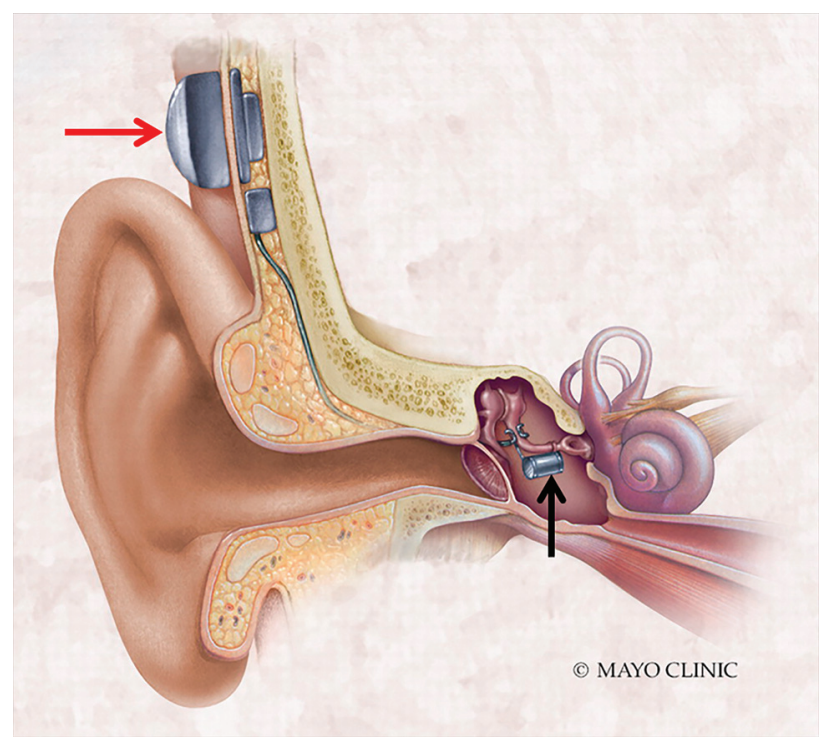

FIG 10. Normal active middle ear implant. Illustration of a partially implantable external audioprocessor (red arrow) attached to the mastoid bone that transmits vibrations to an internal floating mass transducer (black arrow) coupled with the intact ossicular chain. Images used with permission of the Mayo Foundation for Medical Education and Research. All rights reserved.

\section{Jugular Bulb and Sigmoid Sinus Dehiscence Repairs}

Pulsatile tinnitus of venous origin may be caused by abnormalities in the jugular bulb or sigmoid sinus walls leading to jugular bulb or sigmoid sinus dehiscence. ${ }^{31}$ Jugular bulb dehiscence may be seen in patients with a high-riding jugular bulb. Dehiscence can lead to an outpouching of the jugular vein forming a jugular diverticulum into the posterior hypotympanum. A large dehiscent jugular bulb is more common on the right side, where the jugular system is more commonly dominant. Although often asymptomatic, on the basis of the location of jugular diverticulum or dehiscence, symptoms may include conductive hearing loss, pulsatile tinnitus, or brisk venous bleeding after myringotomy. ${ }^{32}$ Reconstruction of jugular bulb dehiscence with hydroxyapatite bone cement or bone pate is an emerging surgical option. ${ }^{33}$ Sigmoid sinus dehiscence may also be seen in patients with idiopathic intracranial hypertension with or without concomitant stenosis at the transverse-sigmoid sinus junction and the more laterally placed sigmoid sinus. ${ }^{34}$ Recently, transmastoid repair for sigmoid sinus dehiscence has been described. This comprises a cortical mastoidectomy, which is further extended to the lateral wall of the sigmoid sinus. The dehiscent sigmoid sinus wall is then reconstructed with bone 
pate or bone cement. Other less preferred reconstruction materials include autologous bone graft or temporalis fascia. ${ }^{31}$ On imaging, it is important to recognize both pre- and postoperative findings to avoid misdiagnosis (Fig 13). Postoperative imaging should also be scrutinized for potential complications such as deep cerebral venous thrombosis and mass effect on the sigmoid sinus or jugular bulb. ${ }^{31}$

\section{Petrous Apicectomy}

The petrous apex is anatomically complex and one of the least accessible areas of the skull base. It is prone to a variety of pathologies

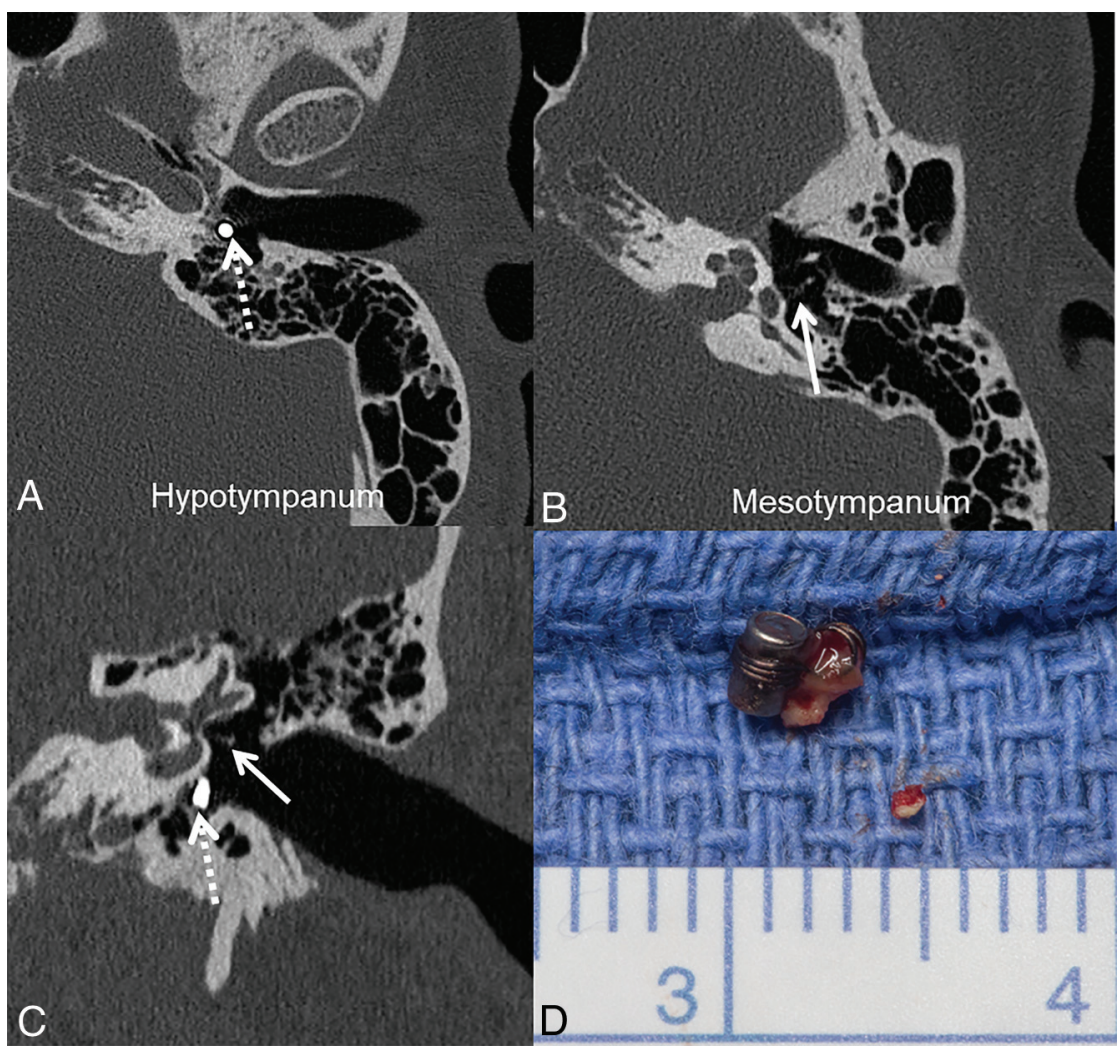

FIG 11. Dislodged floating mass transducer in a partially implantable active middle ear implant. Axial (A) and coronal (C) CT images show a dislodged floating mass transducer lying in the hypotympanum (dotted white arrows) instead of being attached to the stapes in the mesotympanum (solid white arrows, $B, C)$. Postoperative photograph $(D)$ shows the surgically retrieved floating mass transducer. such as petrous apicitis, cholesterol granuloma, epidermoid, mucocele, and tumors. ${ }^{35}$ Surgical drainage of the petrous apex is considered in the setting of locally compressive symptoms or in petrous apicitis with Gradenigo syndrome (triad of retro-orbital pain, otorrhea, and abducens nerve palsy). ${ }^{35,36}$ Multiple surgical approaches have been described reflecting the anatomic complexity and the intent of the operation (ie, biopsy, drainage, or removal) and to safeguard the various vascular and neural structures in this region. The 3 most commonly used approaches are transmastoid retrolabyrinthine, transcanal infracochlear, and extended middle cranial fossa. ${ }^{12}$ In the transmastoid infralabyrinthine approach, the surgical path extends below and posterior to the PSC, above the jugular bulb, and behind the facial nerve. In the infracochlear approach, the path extends through the external auditory canal, below the cochlea, and between the petrous carotid canal and jugular bulb. ${ }^{12}$ The role of postoperative imaging is based on the goals of the original option: identification of recurrence if performed for tumor resection or epidermoid, resolution of infection if performed for apicitis, or adequate drainage if performed for cholesterol granuloma (Fig 14).

\section{CONCLUSIONS}

A wide spectrum of postoperative temporal bone findings may be encountered in practice. It is important not to misinterpret several expected postoperative findings for complications, surgical failure, or residual disease. Neuroradiologists' knowledge of the surgical approaches, in addition to associated expected and unexpected postoperative findings, can reduce the risk of misdiagnosis and unnecessary diagnostic testing.

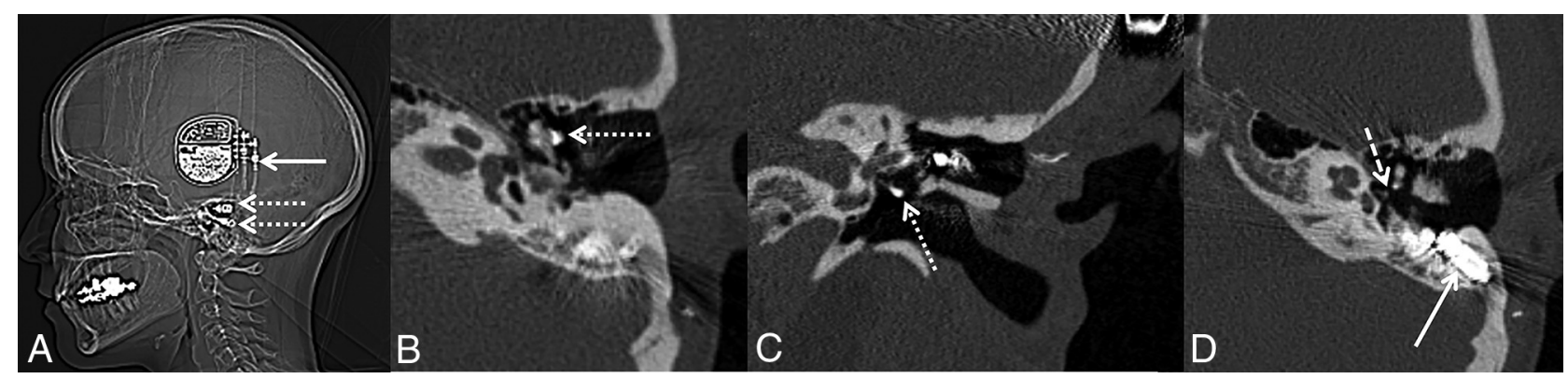

FIG 12. Expected CT findings in totally implantable AMEls. Images show the different components of the totally implantable Esteem AMEI. The CT scout image shows the processor (solid white arrow) and separate internal sensor and driver leads (dotted white arrows). Axial (B and D) and coronal (C) CT images show the sensor on the incudomalleolar articulation (dotted white arrow, B), driver on the stapes (dotted white arrow, $C$ ), and audioprocessor in the mastoid (solid white arrow, D). The incudostapedial joint is disarticulated as part of the surgery (dashed white arrow, D) and should not be misdiagnosed as fracture/failure. 


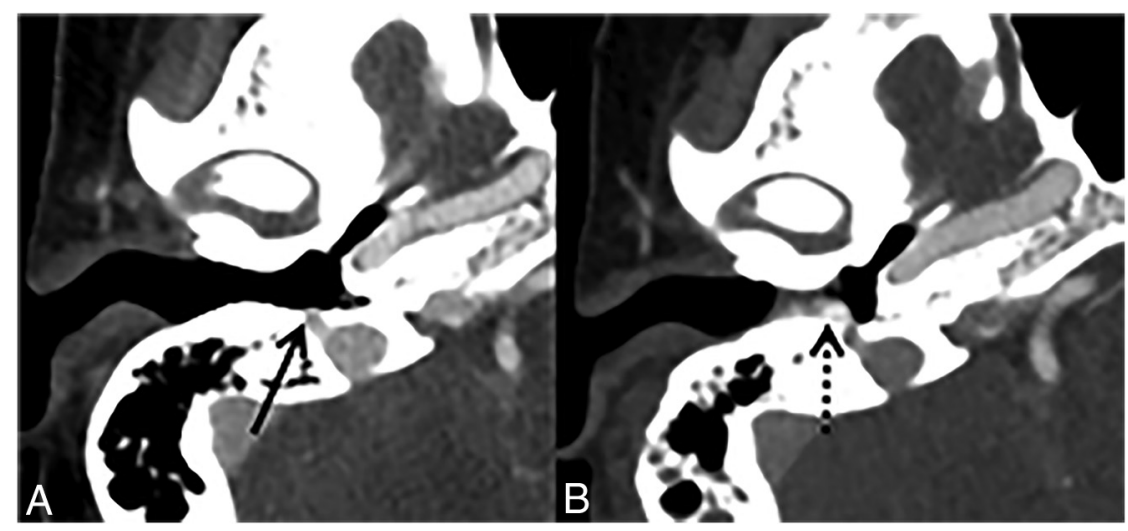

FIG 13. Jugular bulb dehiscence repair. Preoperative $(A)$ and postoperative $(B)$ axial CT images in a patient with pulsatile tinnitus show osseous dehiscence at the lateral margin of the high-riding right jugular bulb, with a diverticulum extending anteriorly to the tympanic annulus (solid black arrow, A). Intraoperatively, a bone pate was harvested from the mastoid cortex and used to cover the high-riding jugular bulb and jugular diverticulum. This is seen as hyperattenuating material at the tympanic annulus and external auditory canal on the postoperative CT (dotted black arrow, $B$ ).

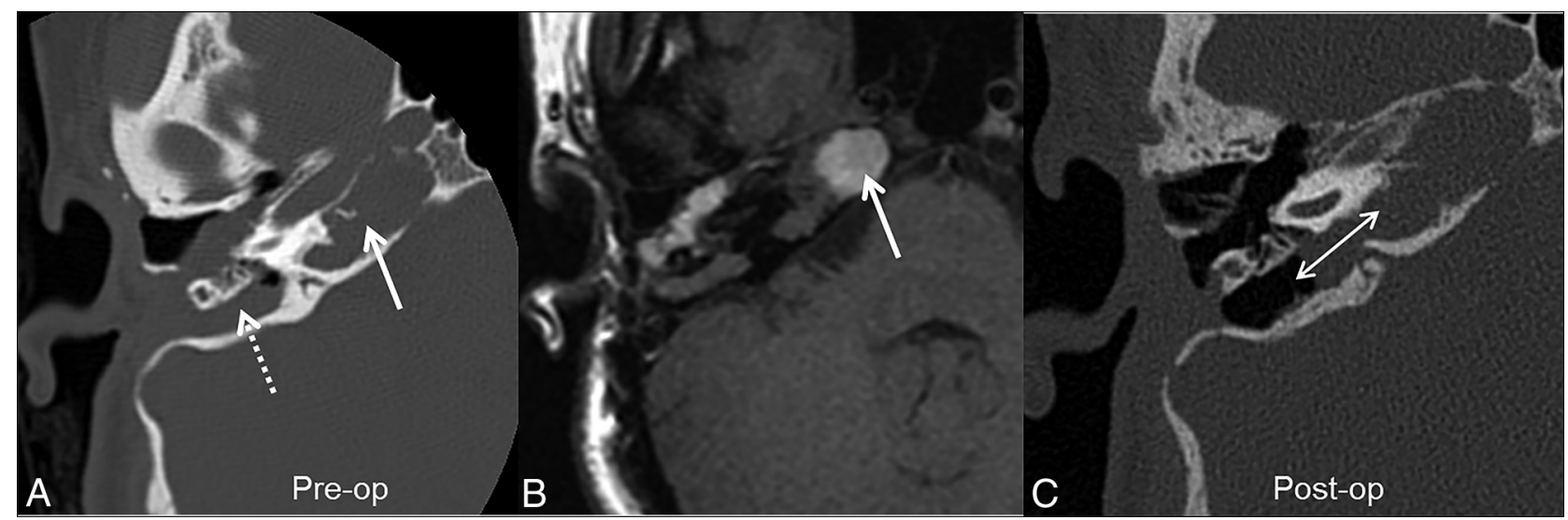

FIG 14. Petrous apex drainage surgery in a patient with recurrent right cholesterol granuloma. Predrainage CT image (A) shows a lesion expanding the petrous apex (solid white arrow) and extending to a prior postoperative mastoid defect (dotted white arrow). On T1-weighted MR imaging $(B)$, the lesion is hyperintense (solid white arrow); it was also T2WI hyperintense and nonenhancing (not shown), consistent with a cholesterol granuloma. C, Postoperative CT image after drainage through a transmastoid infralabyrinthine approach shows the surgically created tract between the petrous and mastoid, allowing drainage of the petrous apex into the mastoid (double-headed white arrow). Pre-op indicates preoperative; Post-op, postoperative.

\section{REFERENCES}

1. Juliano AF, Ginat DT, Moonis G. Imaging review of the temporal bone, Part II: traumatic, postoperative, and noninflammatory nonneoplastic conditions. Radiology 2015;276:655-72 CrossRef Medline

2. Witte RJ, Lane JI, Driscoll CLW, et al. Pediatric and adult cochlear implantation. Radiographics 2003;23:1185-1200 CrossRef Medline

3. Mukherji SK, Mancuso AA, Kotzur IM, et al. CT of the temporal bone: findings after mastoidectomy, ossicular reconstruction, and cochlear implantation. AJR Am J Roentgenol 1994;163:146771 CrossRef Medline

4. Semaan MT, Megerian CA. Meniere's disease: a challenging and relentless disorder. Otolaryngol Clin North Am 2011;44:383-403, ix CrossRef Medline

5. Flores Garcia ML, Llata Segura C, Cisneros Lesser JC, et al. Endolymphatic sac surgery for Meniere's disease: current opinion and literature review. Int Arch Otorhinolaryngol 2017;21:179-83 CrossRef Medline

6. Nevoux J, Barbara M, Dornhoffer J, et al. International consensus (ICON) on treatment of Meniere's disease. Eur Ann Otorhinolaryngol Head Neck Dis 2018;135:S29-32 CrossRef Medline
7. Lo WW, Daniels DL, Chakeres DW, et al. The endolymphatic duct and sac. AJNR Am J Neuroradiol 1997;18:881-87 Medline

8. Kitahara M, Kitano H. Surgical treatment of Meniere's disease. Am J Otol 1985;6:108-09

9. Kitahara T. Evidence of surgical treatments for intractable Meniere's disease. Auris Nasus Larynx 2018;45:393-98 CrossRef Medline

10. Cody DT. Automatic repetitive sacculotomy in endolymphatic hydrops. Otolaryng Clin N Am 1968;1:637-42 CrossRef

11. Roberts DS, Slattery WH. Labyrinthectomy for Meniere's disease. Operative Techniques in Otolaryngology-Head and Neck Surgery 2016;27:188-93 CrossRef

12. Brackmann DE, Shelton C, Arriaga MA. Otologic Surgery. Elsevier; 2016

13. Ho ML. Third window lesions. Neuroimaging Clin N Am 2019;29:5792 CrossRef Medline

14. Moonis G. Imaging of third window lesions of the temporal bone. Semin Roentgenol 2019;54:276-81 CrossRef Medline

15. Weindling SM, Broderick DF. Semicircular canal dehiscence: imaging, diagnosis, classification, surgical options, and postoperative imaging. Neurographics 2016;6:127-37 CrossRef 
16. Mau C, Kamal N, Badeti S, et al. Superior semicircular canal dehiscence: diagnosis and management. J Clin Neurosci 2018;48:58-65 CrossRef Medline

17. Ward BK, Carey JP, Minor LB. Superior canal dehiscence syndrome: lessons from the first 20 years. Front Neurol 2017;8:177 CrossRef Medline

18. Rodgers B, Lin J, Staecker $H$. Transmastoid resurfacing versus middle fossa plugging for repair of superior canal dehiscence: comparison of techniques from a retrospective cohort. World J Otorhinolaryngol Head Neck Surg 2016;2:161-67 CrossRef Medline

19. Succar EF, Manickam PV, Wing S, et al. Round window plugging in the treatment of superior semicircular canal dehiscence. Laryngoscope 2018;128:1445-52 CrossRef Medline

20. Deveze A, Matsuda $H$, Elziere $M$, et al. Diagnosis and treatment of perilymphatic fistula. Adv Otorhinolaryngol 2018;81:133-45 CrossRef Medline

21. Larson TL, Wong ML. Imaging of the mastoid, middle ear, and internal auditory canal after surgery: what every radiologist should know. Neuroimaging Clin N Am 2009;19:307-20 CrossRef Medline

22. Oh MS, Vivas EX, Hudgins PA, et al. The prevalence of superior semicircular canal dehiscence in patients with mastoid encephalocele or cerebrospinal fluid otorrhea. Otol Neurotol 2019;40:485-90 CrossRef Medline

23. Peng T, Ramaswamy AT, Kim AH. Common otologic surgical procedures: clinical decision-making pearls and the role of imaging. Neuroimaging Clin N Am 2019;29:183-96 CrossRef Medline

24. Uezato S, Dias G, Inada J, et al. Imaging aspects of CamuratiEngelmann disease. Rev Assoc Med Bras (1992) 2016;62:825-27 CrossRef Medline

25. Andreu-Arasa VC, Sung EK, Fujita A, et al. Otosclerosis and dysplasias of the temporal bone. Neuroimaging Clin N Am 2019;29:29-47 CrossRef Medline
26. Svrakic M, Vambutas A. Medical and audiological indications for implantable auditory devices. Otolaryngol Clin North Am 2019;52:195210 CrossRef Medline

27. Pok SM, Schlogel M, Boheim K. Clinical experience with the active middle ear implant Vibrant Soundbridge in sensorineural hearing loss. Adv Otorhinolaryngol 2010;69:51-58 CrossRef Medline

28. Loney EL. The role of radiology in active middle ear implantation. Clin Radiol 2014;69:e323-30 CrossRef Medline

29. Labassi S, Beliaeff M, Pean V, et al. The Vibrant Soundbridge middle ear implant: a historical overview. Cochlear Implants Int 2017;18:31423 CrossRef Medline

30. Seidman MD, Janz TA, Shohet JA. Totally implantable active middle ear implants. Otolaryngol Clin North Am 2019;52:297-309 CrossRef Medline

31. Raghavan P, Serulle Y, Gandhi D, et al. Postoperative imaging findings following sigmoid sinus wall reconstruction for pulse synchronous tinnitus. AJNR Am J Neuroradiol 2016;37:136-42 CrossRef Medline

32. Manjila S, Bazil T, Kay M, et al. Jugular bulb and skull base pathologies: proposal for a novel classification system for jugular bulb positions and microsurgical implications. Neurosurg Focus 2018;45: E5 CrossRef Medline

33. DeHart AN, Shaia WT, Coelho DH. Hydroxyapatite cement resurfacing the dehiscent jugular bulb: novel treatment for pulsatile tinnitus. Laryngoscope 2018;128:1186-90 CrossRef Medline

34. Lansley JA, Tucker W, Eriksen MR, et al. Sigmoid sinus diverticulum, dehiscence, and venous sinus stenosis: potential causes of pulsatile tinnitus in patients with idiopathic intracranial hypertension? AJNR Am J Neuroradiol 2017;38:1783-88 CrossRef Medline

35. Razek AA, Huang BY. Lesions of the petrous apex: classification and findings at CT and MR imaging. Radiographics 2012;32:151-73 CrossRef Medline

36. Chapman PR, Shah R, Curé JK, et al. Petrous apex lesions: pictorial review. AJR Am J Roentgenol 2011;196:WS26-37 CrossRef Medline 